\title{
含二氟甲基取代的吡唑酰胺类化合物的合成及杀菌活性
}

\author{
刘婷婷 $a$ 倪 芸 ${ }^{a}$ 钟良坤 ${ }^{a}$ 黄红英 ${ }^{b}$ \\ 胡伟群 $b$ 许天明 $*, b$ 谭成侠 $*, a$ \\ ( ${ }^{a}$ 浙江工业大学化学工程学院 杭州 310014) \\ $\left({ }^{b}\right.$ 中化蓝天集团有限公司 浙江省化工研究院有限公司 杭州 310023)
}

\begin{abstract}
摘要 为了寻找高效安全的杀菌剂新品种和具有生物活性的新型先导化合物, 根据活性亚结构拼接原理, 将异噁唑和 含氟基团引入到吡唑酰胺结构中, 设计了 13 个未见文献报道的含异噁唑的二氟甲基吡唑酰胺类化合物, 通过 ${ }^{1} \mathrm{H} \mathrm{NMR,}$ MS 和 HRMS 对其结构进行了表征, 并对目标产物进行了初步的生物活性测定. 结果表明, 在剂量为 $200 \mathrm{mg} / \mathrm{L}$ 时, 大部 分化合物均表现出较高的杀菌活性.
\end{abstract}

关键词 异噁坐; 吡唑酰胺; 合成; 杀菌活性

\section{Synthesis and Fungicidal Activity of Difluoromethy Substituted Carboxamide Derivatives}

\author{
Liu, Tingting ${ }^{a}$ \\ Ni, Yun ${ }^{a} \quad$ Zhong, Liangkun ${ }^{a}$ \\ Huang, Hongying ${ }^{b}$ \\ $\mathrm{Hu}, \mathrm{Weiqun}^{b}$ \\ $\mathrm{Xu}$, Tianming ${ }^{*, b}$ \\ Tan, Chengxia ${ }^{*, a}$ \\ ( ${ }^{a}$ College of Chemical Engineering, Zhejiang University of Technology, Hangzhou 310014) \\ ( ${ }^{b}$ Sinochem Lantian Zhejiang Research Institute of Chemical Industry Co. Ltd, Hangzhou 310023)
}

\begin{abstract}
In the course of discovering novel fungicidal lead compounds with high activity and safety, 13 new difluoromethyl substituted carboxamide derivatives were designed and synthesized according to the principle of bioactive substructure combination. Their structures were confirmed by ${ }^{1} \mathrm{H}$ NMR, MS and HRMS. The fungicidal activities of these compounds were evaluated. Preliminary bioassay showed that most of the title compounds showed good fungicidal activity at $200 \mathrm{mg} / \mathrm{L}$.
\end{abstract}

Keywords isoxazole; pyrazole amides; synthesis; fungicidal activity

杂环化合物由于其多变的结构和广泛的生物活性 使得在农药的开发中受到广泛的关注 ${ }^{[14]}$, 尤其是含氮 杂环化合物. 吡唑酰胺类化合物作用机理独特、低毒, 吡唑环上的取代位点多, 取代位点的不同和取代基的不 同使该类化合物具有不同的生物活性，已经成为农药研 究的热点之一 ${ }^{[5,6]}$. 当吡唑 4 位上为酰胺取代基时往往表 现出杀菌活性 ${ }^{[7]}$, 其中含氟吡唑酰胺化合物由于结构新 顷、广谱高效和不同于现有杀菌剂的作用方式, 俨然预 示着杀菌剂开发的又一个新热点 ${ }^{[8]}$. 最新报道的杀菌剂 中有 4 个都是以 3 -二氟甲基- $1 H$-甲基吡唑-4-羧酸为中间 体合成的具有良好生物活性的吡唑酰胺化合物, 拜耳公
司开发的吡唑酰胺杀菌剂 Bixafen, 先正达发现并开发 的 Isopyrazam, 巴斯夫公司公布的化合物 fluxapyroxad 和先正达处于研究开发后期的另一个二氟甲基吡唑酰 胺类杀菌剂 Sedaxane (Scheme 1).

异噁唑化合物具有低毒、高效等优良的生物活性, 将异噁唑引入到某些活性分子中, 则可明显提高其生物 活性 ${ }^{[9,10]}$ 。通过 1,3-偶极子与不饱和烃发生 1,3-偶极环加 成将异噁唑环引入, 方法简单易行.

鉴于大部分专利在吡唑酰胺和末端芳杂环之间以 芳环或者碳原子作为桥键 ${ }^{[11 ~ 14]}$, 我们考虑以 3-二氟甲 基- $1 H$-甲基吡唑-4-羧酸为中间体，在吡唑酰胺和芳环之

*E-mail: tanchengxia@zjut.edu.cn

Received June 13, 2014; revised September 22, 2014; published online October 22, 2014

Supported by the Key Innovation Team of Science and Technology in Zhejiang Province (No. 2010R50018), and the Project supported by the National Key Technologies R\&D Program (No. 2011BAE06B03-01).

“十二五” 国家科技支撑计划(No. 2011BAE06B03-01)、浙江省重点科技创新团队(No. 2010R50018)资助项目. 
<smiles>Cn1cc(C(=O)Nc2ccc(F)cc2-c2ccc(Cl)c(Cl)c2)c(C(F)F)n1</smiles>

bixafen<smiles>Cn1cc(C(=O)Nc2ccccc2C2CC2C2CC2)c(C(F)F)n1</smiles><smiles>COc1ccccc1-c1cc(F)c(F)c(F)c1</smiles>

图式 1 最新报道的 4 个吡唑酰胺杀菌剂

Scheme 1 New report of 4 pyrazolecarboaximide fungicides 间引入具有广泛生物活性的含亚甲基的异啞唑杂环，同 时对末端芳基进行修饰. 合成 13 个未见报道的含二氟 甲基吡唑酰胺化合物, 并对它们进行了核磁共振、质谱 的表征, 测试了它们的杀菌活性, 发现在 $200 \mathrm{mg} / \mathrm{L}$ 浓度 下，该类化合物对黄瓜霜霉病和黄瓜白粉病均有较好的 抑制活性. 目标化合物的演化路线如 Eq. 1 所示. 具体 合成路线如 Scheme 2 所示, 化合物列表见表 1.<smiles>Cn1cc(C(=O)NC2CC3CC(C(F)F)CC(C3)C2(F)F)c(C(F)F)n1</smiles>

表 1 化合物 12a $\sim 12 \mathrm{n}$ 一览表

Table 1 Structures of products $12 \mathrm{a} \sim 12 \mathrm{~m}$

\begin{tabular}{cl||cl}
\hline Compd. & \multicolumn{1}{c||}{$\mathrm{Ar}$} & Compd. & \multicolumn{1}{c}{$\mathrm{Ar}$} \\
\hline $\mathbf{1 2 a}$ & $2,4,6-\mathrm{Me}_{3} \mathrm{C}_{6} \mathrm{H}_{2}$ & $\mathbf{1 2 h}$ & $2,6-\mathrm{F}_{2} \mathrm{C}_{6} \mathrm{H}_{3}$ \\
$\mathbf{1 2 b}$ & $4-\mathrm{ClC}_{6} \mathrm{H}_{4}$ & $\mathbf{1 2 i}$ & $2,3,4-(\mathrm{MeO})_{3} \mathrm{C}_{6} \mathrm{H}_{2}$ \\
$\mathbf{1 2 c}$ & $2-\mathrm{ClC}_{6} \mathrm{H}_{4}$ & $\mathbf{1 2 j}$ & $2,6-(\mathrm{MeO})_{2} \mathrm{C}_{6} \mathrm{H}_{3}$ \\
$\mathbf{1 2 d}$ & $4-\mathrm{MeOC}_{6} \mathrm{H}_{4}$ & $\mathbf{1 2 k}$ & $2-\mathrm{HOC}{ }_{6} \mathrm{H}_{4}$ \\
$\mathbf{1 2 e}$ & $4-\mathrm{Me}_{2} \mathrm{CHC}_{6} \mathrm{H}_{4}$ & $\mathbf{1 2 l}$ & $4-(\mathrm{Me})_{3} \mathrm{CC}_{6} \mathrm{H}_{4}$ \\
$\mathbf{1 2 f}$ & $4-N, N-\mathrm{Me}_{2} \mathrm{NC}_{6} \mathrm{H}_{4}$ & $\mathbf{1 2 m}$ & $2-\mathrm{Me}-$ thienyl \\
$\mathbf{1 2 g}$ & $2-\mathrm{F}-6-\mathrm{ClC}_{6} \mathrm{H}_{3}$ & & \\
\hline
\end{tabular}

\section{1 结果与讨论}

1.1 中间体 3-二氟甲基-1H-甲基吡唑-4-羧酸和目标化 合物的合成

3-二氟甲基- $1 H$-甲基吡唑-4-羧酸作为一个重要的 农药中间体, 合成方法较多, 也比较成熟. 本文参考先 正达公司的专利 ${ }^{[15]}$ 的合成方法, 以二氟乙酰乙酸乙酯 和原甲酸三乙酯为起始原料, 在乙酸酐中反应得到 2-乙 氧甲叉基-4,4-二氟乙酸乙酯, 再在碱参与下滴加到甲基
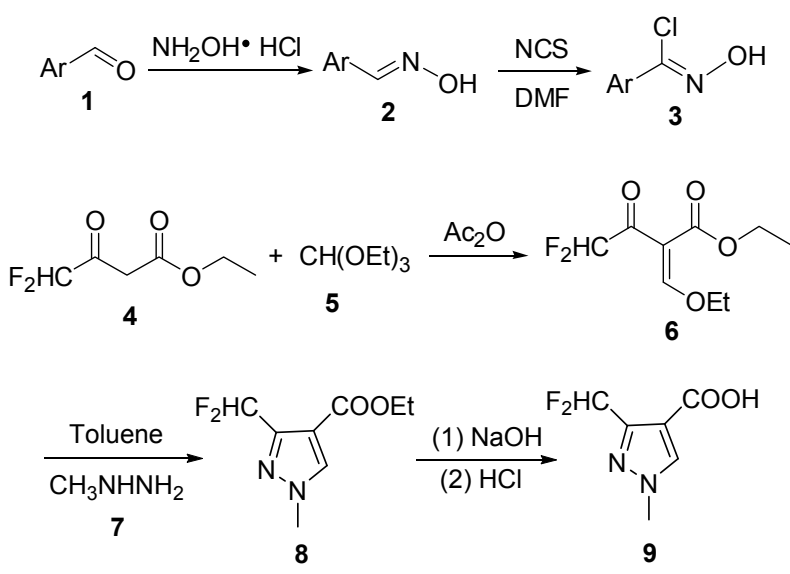<smiles>C#CCNC(=O)c1cn([Tl])nc1C(F)F</smiles><smiles>[CH2]C(C)/C(Cl)=N/O</smiles><smiles>Cn1cc(C(=O)NCc2cc([Al])no2)c(C(F)F)n1</smiles>

图式 2 目标化合物合成路线

Scheme 2 Synthesis route of target compounds

肼中, 最后碱性条件下水解得到 3-二氟甲基- $1 H$-甲基吡 唑-4-羧酸. 不同溶剂的选择对于反应收率和纯度有一 定影响, 通过表 2 可以看出, 综合考虑成本、产率和纯 度，选择甲苯作溶剂。

表 2 溶剂对反应的影响

Table 2 The effect of various solvents on the reaction

\begin{tabular}{clcc}
\hline 编号 & 溶剂 & 产率\% & 主产物 : 副产物 \\
\hline 1 & 二甲苯 & 94 & $97: 3$ \\
2 & 甲苯 & 94 & $97: 3$ \\
3 & 氯苯 & 87 & $95: 5$ \\
4 & 环己烷 & 86 & $91: 9$ \\
5 & 二氧六环 & 78 & $91: 9$ \\
\hline
\end{tabular}

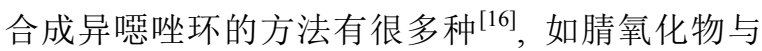
不饱和键的偶极环加成反应，差胺与 $\beta$-不饱和酮或酯的 反应，分子内羟肜与不饱和键的加成反应等. 结合本实 验的条件, 在合成中用氯代丁二酰亚胺(NCS)将醛肟转 化为氯代肜，然后用三乙胺处理，生成腈氧化物，再与 
炔烃发生 1,3-偶极环加成反应得到异噁唑环(Scheme 3). 此方法条件温和, 在室温下即可发生偶极反应生成异噁 唑环.
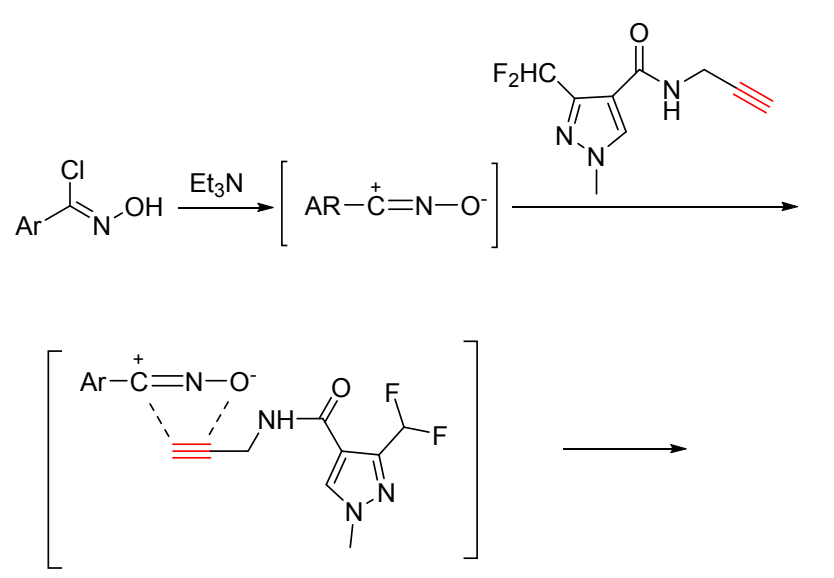<smiles>Cn1cc(C(=O)NCc2cc(Br)no2)c(C(F)F)n1</smiles>

图式 3 异朢唑的合成机理

Scheme 3 Synthetic mechanism of isoxazole

在 ${ }^{1} \mathrm{H}$ NMR 谱中, 吡唑环上的二氟甲基由于诱导效 应, 基团上的氢原子的共振峰移向低场, 同一个碳原子 上的两个氟原子对氢有偶合作用, 形成 $\mathrm{t}$ 峰, 且氟原子 对氢原子的偶合较大, 偶合常数为 $36.0 \mathrm{~Hz}$, 此峰为特征 峰, 一般在 $\delta 6.89$ 附近. 吡唑环上的氢受到屏蔽作用, 谱峰出现在低场, 在 $\delta 7.95$ 附近出现一个尖锐的 $\mathrm{s}$ 峰, 吡唑环上的甲基由于氮原子的屏蔽作用, $\mathrm{s}$ 峰的化学位 移在 $\delta 3.94$ 左右; $\mathrm{CONH}$ 中由于受到氮的电负性的影响, 使得酰胺基上的氢峰形成宽峰, 化学位置在 $\delta 6.50$ 附近. 桥键亚甲基上的氢由于酰胺上的氢原子的偶合作用, 裂 分为三重峰, $\mathrm{t}$ 峰化学位移在 $\delta 4.80$ 左右, 异噁唑环的电 子云易受到与它相连的芳环取代基的影响, 导致异噁唑 上氢原子的 s 峰出现在 $\delta 6.10 \sim 6.50$ 附近.

\section{2 杀菌活性}

供试菌株为黄瓜灰霉病菌(Botrytis cinerea)、黄瓜霜 霉病菌(Bremia lactucae)和黄瓜白粉病菌(Erysiphaceae). 以上菌种均保存在冰箱 $\left(4 \sim 8{ }^{\circ} \mathrm{C}\right)$ 内, 试验前 $2 \sim 3 \mathrm{~d}$ 从 试管斜面接种到培养皿中, 在适宜的温度下培养供试验 用. 试验作物为黄瓜, 将盆栽黄瓜苗培养至 2 叶 1 心期, 供试验用。

参照《国家南方农药创制中心生测标准程序》 ${ }^{[17]}$, 准确称取(精确至 $0.0001 \mathrm{~g}$ ) 一定质量目标化合物, 加入 含有 $0.1 \%$ 吐温- 80 的 $N, N$-二甲基甲酰胺(DMF), 配制成 化合物质量分数为 $5 \%$ 的母液, 用蒸馏水分别稀释成
$200 \mathrm{mg} / \mathrm{L}$ 质量浓度的药液. 以嘧菌酯原药作阳性对照, 以 DMF 作空白对照.

采用温室活体盆栽法测定, 将试验作物置于喷雾机 上进行叶面喷雾处理, 药剂处理后的作物放置阴凉处, 于 $24 \mathrm{~h}$ 后接种病原菌狍子, 设 3 次重复, 另设空白对照. 接种后的作物置于人工气候室中培养, 对以上病害分别 培养 7 和 $10 \mathrm{~d}$ 后调查防效.

化合物杀菌普篮结果见表 3. 由表 3 可知, 在 200 $\mathrm{mg} / \mathrm{L}$ 浓度下, 该类化合物中, 除 $\mathbf{1 2 m}$ 外对黄瓜霜霉病 和黄瓜白粉病均有较好的抑制活性, 其中 $12 \mathrm{c}, 12 \mathrm{~g}, 12 \mathrm{i}$ 抑制活性达到 $90 \%$ 以上，而该系列对于黄瓜灰霉病无活 性.

表 3 化合物 $12 \mathrm{a} \sim 12 \mathrm{~m}$ 的杀菌活性

Table 3 Fungicidal activity of compounds $12 \mathrm{a} \sim 12 \mathrm{~m}$

\begin{tabular}{|c|c|c|c|}
\hline \multirow[b]{2}{*}{ 化合物 } & \multicolumn{3}{|c|}{ 防效 $/ \%$} \\
\hline & $\begin{array}{c}\text { 黄瓜灰霉病 } \\
\text { (活体) }\end{array}$ & $\begin{array}{c}\text { 黄瓜霜霉病 } \\
\text { (活体) }\end{array}$ & $\begin{array}{c}\text { 黄瓜白粉病 } \\
\text { (活体) }\end{array}$ \\
\hline $12 a$ & 0 & 75 & 80 \\
\hline $12 b$ & 0 & 70 & 85 \\
\hline $12 \mathrm{c}$ & 0 & 65 & 90 \\
\hline 12d & 0 & 50 & 75 \\
\hline $12 \mathrm{e}$ & 0 & 75 & 80 \\
\hline $12 \mathrm{f}$ & 0 & 45 & 65 \\
\hline $12 \mathrm{~g}$ & 0 & 80 & 90 \\
\hline $12 \mathrm{~h}$ & 0 & 50 & 65 \\
\hline $12 \mathrm{i}$ & 0 & 70 & 95 \\
\hline $12 j$ & 0 & 50 & 65 \\
\hline $12 k$ & 0 & 65 & 75 \\
\hline 121 & 0 & 60 & 80 \\
\hline $12 \mathrm{~m}$ & 0 & 0 & 10 \\
\hline 嘧菌酯 & 100 & 100 & 100 \\
\hline
\end{tabular}

\section{2 结论}

本文以二氟乙酰乙酸乙酯为起始原料, 以其作为含 氟砌块参与反应, 制得 3-二氟甲基- $1 H$-甲基吡唑-4-羧酸 中间体, 通过在吡唑环与芳环之间引入具有广泛生物活 性的异噁唑环做桥键, 合成一系列未见报道的含二氟甲 基吡坐酰胺化合物. 生物活性表明, 在 $200 \mathrm{mg} / \mathrm{L}$ 浓度 下, 该类化合物中, 除 $\mathbf{1 2 m}$ 外对黄瓜霜霉病和白粉病均 有较好的抑制活性，其中 $12 \mathrm{c}, 12 \mathrm{~g}, 12 \mathrm{i}$ 抑制活性达到 90\%及以上.

初步构效关系分析知, 3-二氟甲基- $1 H$-甲基吡唑-4甲酰胺可能是活性中心(Scheme 4), 桥键含亚甲基的异 噁唑环对于化合物活性有一定的影响. 取代苯基的化合 物对于黄瓜霜霉病和黄瓜白粉病的活性较好, 对于黄瓜 灰霉病没有活性.

苯环上 R 基团位置和性质的不同, 对化合物杀菌活 性有一定影响, 当苯环上的邻对位有卤素原子取代时, 


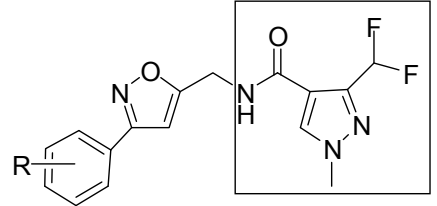

图式 4 化合物生物活性中心

Scheme 4 Biological active center of target compounds

化合物对于黄瓜白粉病的活性更高, 例如, $12 \mathrm{c}, 12 \mathrm{~g}$ 对 于黄瓜白粉病的活性为 $90 \%$.

\section{3 实验部分}

\section{1 仪器与试剂}

核磁共振仪(美国 Varian 500 (TMS 内标)), 质谱仪 (LCQ-Advantage), 旋转蒸发器 IKA-RV10, 熔点测定仪 (北京泰克有限公司 X-4, 温度计未矫正), 恒速搅拌器 (上海申胜生物技术有限公司), MP200B 型电子天平(杭 州万特衡器有限公司), SHB-111A 型循环水式多用真空 原(北京京科瑞达科技有限公司), DLSB-3006 型低温冷 却液循环百(杭州大卫科教仪器有限公司).

乙酸乙酯(上海实验试剂有限公司); 石油醚(济南联 顺化工有限公司); 乙酸䣶(杭州龙山化工有限公司); $N, N$-二甲基甲酰胺(DMF)(济南金贵林化工有限公司); 二氯亚砜(上海海曲化工有限公司); 氢氧化钠(江苏响水 天益化工有限公司); 无水硫酸钠(上海实验试剂有限公 司); 三乙胺 (济南联顺化工有限公司); 四氢呋喃 (THF)(杭州双林化工试剂厂); 二氟乙酰乙酸乙酯(上海 邦成化工有限公司); 甲基肼(上海大业化工有限公司)均 为试剂级.

\section{2 化合物的合成}

\subsection{1 取代甲醛肜 (2)的合成}

以 4-氯苯甲醛肜的合成为例, 其它合成方法相同. 向 $50 \mathrm{~mL}$ 的单口烧瓶中加入 $3 \mathrm{~g}(21 \mathrm{mmol}) 4$-氯苯甲醛, 加入 $10 \mathrm{~mL}$ 无水甲醇溶解, 然后加入 $1.78 \mathrm{~g}(25.6 \mathrm{mmol})$ 盐酸差胺和 $2.23 \mathrm{~g}(21 \mathrm{mmol})$ 碳酸钠, 加热回流 $4 \mathrm{~h}, \mathrm{TLC}$ 检测没有原料点, 将溶剂旋干, 加入 $10 \mathrm{~mL}$ 去离子水, 大量白色固体析出, 抽滤, 水洗, 干燥. 得到 $3.47 \mathrm{~g} 4$-氯 苯甲醛服, 产率 $87 \%$. 其它取代苯甲醛肜合成方法相同.

\section{2 .2 取代苯基氯化肜(3)的合成}

以 4-氯-苯基-1-氯化肜为例, 其它合成方法相同. 向 $50 \mathrm{~mL}$ 的单口烧瓶中加入 $0.3 \mathrm{~g}(1.93 \mathrm{mmol}) 4$-氯苯甲

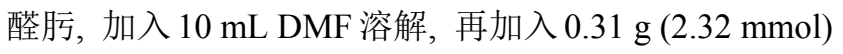
$N$-氯代丁二酰亚胺(NCS), 室温下搅拌 $3 \mathrm{~h}$, TLC 检测没 有原料点. 反应结束后不经处理, 密封保存备用.

\subsubsection{2-乙氧甲叉基-4,4-二氟乙酸乙酯(6)的合成}

参考文献[18]将 $54.78 \mathrm{~g}(0.33 \mathrm{~mol})$ 的二氟乙酰乙酸 乙酯及 $97.68 \mathrm{~g}(0.66 \mathrm{~mol})$ 原甲酸三乙酯和 $70 \mathrm{~mL}$ 乙酸䣶 加入 $250 \mathrm{~mL}$ 反应瓶中, 搅拌下回流 $7 \mathrm{~h}$. 减压蒸去溶剂, 油㬌减压蒸馏, 收集 $98 \sim 105{ }^{\circ} \mathrm{C}$ 馏分, 为黄绿色油状液 体 6, 收率 92.3\%. ${ }^{1} \mathrm{H}$ NMR $\left(400 \mathrm{MHz}, \mathrm{CDCl}_{3}\right) \delta: 1.32 \sim$ $1.39\left(\mathrm{~m}, 3 \mathrm{H}, \mathrm{COCH}_{2} \mathrm{CH}_{3}\right), 1,42 \sim 1.49(\mathrm{~m}, 3 \mathrm{H}, \mathrm{COO}-$ $\left.\mathrm{CH}_{2} \mathrm{CH}_{3}\right), 4.32 \sim 4.39\left(\mathrm{~m}, 4 \mathrm{H}, \mathrm{OCH}_{2} \mathrm{CH}_{3}\right), 6.27 \sim 6.61(\mathrm{~m}$, $1 \mathrm{H},=\mathrm{CH}), 7.91\left(\mathrm{t}, J=52.0 \mathrm{~Hz}, 1 \mathrm{H}, \mathrm{CHF}_{2}\right)$.

\subsubsection{3-二氟甲基- $1 H$-甲基吡唑-4-羧酸(9)的合成}

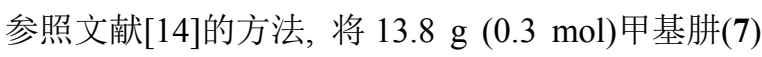
(5\%的水溶液)加入到 $500 \mathrm{~mL}$ 的反应瓶中, 然后将 $8 \mathrm{~g}$ $30 \%$ 的 $\mathrm{NaOH}$ 水溶液加入反应瓶中, 再加入 $75 \mathrm{~g}$ 水, $15^{\circ} \mathrm{C}$ 下搅拌, 将 $66.6 \mathrm{~g}(0.3 \mathrm{~mol})$ 的 2-乙氧甲叉基-4,4-二 氟乙酸乙酯(6)溶于 $85 \mathrm{~g}$ 甲苯中, 在 $15{ }^{\circ} \mathrm{C}$ 下将甲苯溶液 滴加到反应瓶中, $30 \mathrm{~min}$ 滴完, 将温度升到 $25{ }^{\circ} \mathrm{C}$ 继续摚 拌 $15 \mathrm{~min}, 65{ }^{\circ} \mathrm{C}$ 下向反应瓶中加入 $60 \mathrm{~g} \mathrm{30 \%}$ 的 $\mathrm{NaOH}$ 水 溶液, 搅拌 $45 \mathrm{~min}$. 停止搅拌, 趁热分液取甲苯层. 向 甲苯层中加入 $50 \mathrm{~g}$ 水和 $65 \mathrm{~g} \mathrm{32 \%}$ 的 $\mathrm{HCl}$ 溶液, 加热至 $95^{\circ} \mathrm{C}$ 搅拌 $10 \mathrm{~min}$. 将溶液冷却至室温 $\left(25^{\circ} \mathrm{C}\right)$, 有大量固 体析出, 过滤, 滤饼用去离子水洗涤 2 次, 烘干, 得到白 色粉末状固体，产率 83.6\%. ${ }^{1} \mathrm{H}$ NMR $(400 \mathrm{MHz}$, DMSO- $\left.d_{6}\right) \delta: 3.91\left(\mathrm{~s}, 3 \mathrm{H}, \mathrm{CH}_{3}\right), 7.19(\mathrm{t}, J=54.0 \mathrm{~Hz}, 1 \mathrm{H}$, $\left.\mathrm{CHF}_{2}\right), 8.31(\mathrm{~s}, 1 \mathrm{H}$, pyrazole-H), $12.73(\mathrm{~s}, 1 \mathrm{H}, \mathrm{COOH})$.

3.2.5 3-二氟甲基- $1 H$-甲基吡唑-4-基-丙炔甲酰胺 (11)的合成

向 $100 \mathrm{~mL}$ 单口烧瓶中加入 $18.44 \mathrm{~g}(0.105 \mathrm{~mol}) 3-$ 二 氟甲基- $1 \mathrm{H}$-甲基吡唑-4-羧酸和 $74.8 \mathrm{~g}(0.62 \mathrm{~mol}) \mathrm{SOCl}_{2}$, 加热回流 $4 \mathrm{~h}$. 反应结束后, 常压蒸馏出去多余二氯亚 砜, 得到红色液体 10, 不经处理, 密封保存待用.

向 $500 \mathrm{~mL}$ 三口烧瓶中加 $12.9 \mathrm{~g}(66 \mathrm{mmol})$ 上述酰氯 10, 加入 $100 \mathrm{~mL}$ 四氢呋喃, 然后在 $10{ }^{\circ} \mathrm{C}$ 下将 $3.65 \mathrm{~g}(66$ $\mathrm{mmol}$ )炔丙胺和 $10.05 \mathrm{~g}$ 三乙胺混合液缓慢滴加到反应 瓶中, $1 \mathrm{~h}$ 滴加完毕, 升温至 $25{ }^{\circ} \mathrm{C}$ 继续搅拌 $1 \mathrm{~h}$. 过滤, 滤液脱溶, 得到深红色油状液体. ${ }^{1} \mathrm{H}$ NMR $(400 \mathrm{MHz}$, $\left.\mathrm{CDCl}_{3}\right) \delta: 2.27$ (s, $\left.1 \mathrm{H}, \mathrm{CH}\right), 3.93\left(\mathrm{~s}, 3 \mathrm{H}, \mathrm{CH}_{3}\right), 4.20(\mathrm{~s}, 2 \mathrm{H}$, $\left.\mathrm{CH}_{2}\right), 6.54(\mathrm{~s}, 1 \mathrm{H}, \mathrm{CONH}), 7.00(\mathrm{t}, J=28.0 \mathrm{~Hz}, 1 \mathrm{H}$, $\mathrm{CHF}_{2}$ ), 7.94 (s, 1H, pyrazole-H).

\subsection{6 目标化合物 $12 \mathrm{a} \sim 12 \mathrm{p}$ 合成}

向对氯苯基氯化肜(3)的反应瓶中加入 $0.41 \mathrm{~g}$ (1.93 $\mathrm{mmol}$ )化合物 11 , 再加入 $0.29 \mathrm{~g}$ 三乙胺 $(2.89 \mathrm{mmol})$, 室 温下搅拌 $5 \mathrm{~h}$. TCL 检测无原料点. 向反应瓶中加入 20 $\mathrm{mL}$ 去离子水, 用乙酸乙酯萃取, 有机相用去离子水洗 涤两次, 脱溶, 柱层析石油醚/乙酸乙酯(体积比 $5: 1$ )得 目标化合物 12b, 其它 15 个化合物的合成方法相同. 
3-二氟甲基- $N$-[(3-均三甲苯异噁唑-5-基)-甲基]-1甲基- $1 H$-吡唑-4-甲酰胺(12a): 白色粉末，产率 50.8\%. m.p. $47 \sim 49{ }^{\circ} \mathrm{C} ;{ }^{1} \mathrm{H}$ NMR $\left(\mathrm{CDCl}_{3}, 400 \mathrm{MHz}\right) \delta$ : 2.12 (s, $\left.6 \mathrm{H}, \mathrm{Ar}-\mathrm{CH}_{3}\right), 2.31$ (s, 3H, Ar- $\left.\mathrm{CH}_{3}\right), 3.29$ (s, 3H, pyrazole- $\left.\mathrm{CH}_{3}\right), 4.81\left(\mathrm{~d}, J=8.0 \mathrm{~Hz}, 2 \mathrm{H}, \mathrm{CH}_{2}\right), 6.16(\mathrm{~s}, 1 \mathrm{H}$, isoxazole-H), $6.71 \sim 6.86(\mathrm{~m}, 3 \mathrm{H}, \mathrm{Ar}-\mathrm{H}$ and $\mathrm{CONH}), 6.92$ (t, $\left.J=24.0 \mathrm{~Hz}, 1 \mathrm{H}, \mathrm{CHF}_{2}\right), 7.96$ (s, $1 \mathrm{H}$, pyrazole-H); MS (ESI) $m / z: 375[\mathrm{M}+\mathrm{H}]^{+}$; HRMS calcd for $\mathrm{C}_{19} \mathrm{H}_{21} \mathrm{~F}_{2} \mathrm{~N}_{4} \mathrm{O}_{2}$ 375.1627, found 375.1637.

3-二氟甲基- $N$-[(3-(4-氯苯基)异噁唑-5-基)-甲基]-1甲基- $1 H$ - 吡唑-4-甲酰胺(12b): 黄色油状液体, 产率 66.4\%. ${ }^{1} \mathrm{H}$ NMR $\left(\mathrm{CDCl}_{3}, 400 \mathrm{MHz}\right) \delta: 3.37$ (s, 3H, pyrazole- $\left.\mathrm{CH}_{3}\right), 4.76\left(\mathrm{~d}, J=8.0 \mathrm{~Hz}, 2 \mathrm{H}, \mathrm{CH}_{2}\right), 6.52(\mathrm{~s}, 1 \mathrm{H}$, isoxazole-H), $6.58(\mathrm{~s}, 1 \mathrm{H}, \mathrm{CONH}), 6.90(\mathrm{t}, J=52.0 \mathrm{~Hz}$, $1 \mathrm{H}, \mathrm{CHF}_{2}$ ), 7.42 (d, $\left.J=8.0 \mathrm{~Hz}, 2 \mathrm{H}, \mathrm{Ar}-\mathrm{H}\right), 7.71$ (d, $J=8.0$ $\mathrm{Hz}, 2 \mathrm{H}, \mathrm{Ar}-\mathrm{H}), 7.99$ (s, 1H, pyrazole-H); MS (ESI) $m / z$ : $367[\mathrm{M}+\mathrm{H}]^{+}$. HRMS calcd for $\mathrm{C}_{16} \mathrm{H}_{13} \mathrm{ClF}_{2} \mathrm{~N}_{4} \mathrm{O}_{2} 367.0768$, found 367.0771 .

3-二氟甲基- $N$-[(3-(2-氯苯基)异硻唑-5-基)-甲基]1-甲基- $1 H$-吡唑-4-甲酰胺(12c): 黄色油状液体, 产率 72.4\%. ${ }^{1} \mathrm{H}$ NMR $\left(\mathrm{CDCl}_{3}, 400 \mathrm{MHz}\right) \delta: 3.94(\mathrm{~s}, 3 \mathrm{H}$, pyrazole- $\left.\mathrm{CH}_{3}\right), 4.81\left(\mathrm{~d}, J=8.0 \mathrm{~Hz}, 2 \mathrm{H}, \mathrm{CH}_{2}\right), 6.48(\mathrm{~s}, 1 \mathrm{H}$, CONH), $6.70(\mathrm{~s}, 1 \mathrm{H}$, isoxazole-H), $6.85(\mathrm{t}, J=36.0 \mathrm{~Hz}$, $\left.1 \mathrm{H}, \mathrm{CHF}_{2}\right), 7.33 \sim 7.40(\mathrm{~m}, 2 \mathrm{H}, \mathrm{Ar}-\mathrm{H}), 7.48(\mathrm{~d}, J=8.0 \mathrm{~Hz}$, 1H, Ar-H), 7.71 (d, $J=8.0 \mathrm{~Hz}, 1 \mathrm{H}, \operatorname{Ar}-\mathrm{H}), 7.97$ (s, 1H, pyrazole-H); MS (ESI) $m / z: 367[\mathrm{M}+\mathrm{H}]^{+}$. HRMS calcd for $\mathrm{C}_{16} \mathrm{H}_{13} \mathrm{ClF}_{2} \mathrm{~N}_{4} \mathrm{O}_{2} 367.0768$, found 367.0786 .

3 -二氟甲基- $N$-[(3-(4-甲氧基苯基)异噁唑-5-基)-甲 基]-1-甲基-1 $H$-吡唑-4-甲酰胺(12d)：黄色油状液体，产 率 45.7\%. ${ }^{1} \mathrm{H}$ NMR $\left(\mathrm{CDCl}_{3}, 400 \mathrm{MHz}\right) \delta: 3.85$ (s, 3H, $\left.\mathrm{OCH}_{3}\right), 3.93\left(\mathrm{~s}, 3 \mathrm{H}\right.$, pyrazole- $\left.\mathrm{CH}_{3}\right), 4.49(\mathrm{~d}, J=8.0 \mathrm{~Hz}$, $\left.2 \mathrm{H}, \mathrm{CH}_{2}\right), 6.49(\mathrm{~s}, 1 \mathrm{H}$, isoxazole-H), $6.55(\mathrm{~s}, 1 \mathrm{H}, \mathrm{CONH})$, 6.89 (t, $\left.J=36.0 \mathrm{~Hz}, 1 \mathrm{H}, \mathrm{CHF}_{2}\right), 6.94 \sim 6.97(\mathrm{~m}, 2 \mathrm{H}$, Ar-H), 7.71 (d, $J=8.0 \mathrm{~Hz}, 2 \mathrm{H}$, Ar-H), 7.97 (s, 1H, pyrazole-H); MS (ESI) $m / z: 363[\mathrm{M}+\mathrm{H}]^{+}$. HRMS calcd for $\mathrm{C}_{17} \mathrm{H}_{16} \mathrm{~F}_{2} \mathrm{~N}_{4} \mathrm{O}_{3} 363.1263$, found 363.1279 .

3 -二氟甲基- $N$-[(3-(4-异丙基苯基)异噁唑-5-基)-甲 基]-1-甲基- $1 H$-吡唑-4-甲酰胺(12e): 黄色油状液体, 产 率 78.9\%. ${ }^{1} \mathrm{H}$ NMR $\left(\mathrm{CDCl}_{3}, 400 \mathrm{MHz}\right) \delta: 1.28[\mathrm{~s}, 6 \mathrm{H}$, $\left.\mathrm{CH}\left(\mathrm{CH}_{3}\right)_{2}\right], 2.05 \quad\left[\mathrm{~s}, \quad 1 \mathrm{H}, \quad \mathrm{CH}\left(\mathrm{CH}_{3}\right)_{2}\right], 3.93 \quad(\mathrm{~s}, 3 \mathrm{H}$, pyrazole- $\left.\mathrm{CH}_{3}\right), 4.76\left(\mathrm{~d}, J=8.0 \mathrm{~Hz}, 2 \mathrm{H}, \mathrm{CH}_{2}\right), 6.52(\mathrm{~s}, 1 \mathrm{H}$, isoxazole-H), $6.55(\mathrm{~s}, 1 \mathrm{H}, \mathrm{CONH}), 6.89(\mathrm{t}, J=36.0 \mathrm{~Hz}$, $1 \mathrm{H}, \mathrm{CHF}_{2}$ ), 7.30 (d, $\left.J=8.0 \mathrm{~Hz}, 2 \mathrm{H}, \mathrm{Ar}-\mathrm{H}\right), 7.70$ (d, $J=8.0$ $\mathrm{Hz}, 2 \mathrm{H}, \mathrm{Ar}-\mathrm{H}$ ), 7.97 (s, 1H, pyrazole-H); MS (ESI) $m / z$ : $375[\mathrm{M}+\mathrm{H}]^{+}$. HRMS calcd for $\mathrm{C}_{19} \mathrm{H}_{21} \mathrm{~F}_{2} \mathrm{~N}_{4} \mathrm{O}_{2}$ 375.1627, found 375.1640 .

3-二氟甲基- $N$-[(3-(4-(二甲氨基)苯基)异噁唑-1基)-甲基]-5-甲基- $1 \mathrm{H}$-吡唑-4-甲酰胺(12f)：黄色油状液 体, 产率 44.4\%. ${ }^{1} \mathrm{H} \mathrm{NMR}\left(\mathrm{CDCl}_{3}, 400 \mathrm{MHz}\right) \delta$ : 3.02 (s, $\left.6 \mathrm{H}, \mathrm{N}\left(\mathrm{CH}_{3}\right)_{2}\right), 3.94$ (s, $3 \mathrm{H}$, pyrazole- $\left.\mathrm{CH}_{3}\right), 4.74(\mathrm{~d}, J=8.0$ $\left.\mathrm{Hz}, 2 \mathrm{H}, \mathrm{CH}_{2}\right), 6.47(\mathrm{~s}, 1 \mathrm{H}$, isoxazole- $\mathrm{H}), 6.73 \sim 6.82(\mathrm{~m}$, $3 \mathrm{H}, \mathrm{Ar}-\mathrm{H}$ and $\mathrm{CONH}), 6.87\left(\mathrm{~d}, J=36.0 \mathrm{~Hz}, 1 \mathrm{H}, \mathrm{CHF}_{2}\right.$ ), 7.66 (d, $J=8.0 \mathrm{~Hz}, 2 \mathrm{H}, \mathrm{Ar}-\mathrm{H}), 7.96$ (s, 1H, pyrazole-H); MS (ESI) $m / z: 376[\mathrm{M}+\mathrm{H}]^{+}$. HRMS calcd for $\mathrm{C}_{18} \mathrm{H}_{19} \mathrm{~F}_{2} \mathrm{~N}_{5} \mathrm{O}_{2} 376.1580$, found 376.1588 .

3 -二氟甲基- $N-[(3-(2$-氯- 6 -氟苯基)异噁唑-5-基)-甲 基]-1-甲基- $1 H$-吡唑-4-甲酰胺(12g)：黄色油状液体，产 率 38.9\%. ${ }^{1} \mathrm{H}$ NMR $\left(\mathrm{CDCl}_{3}, 400 \mathrm{MHz}\right) \delta: 3.94(\mathrm{~s}, 3 \mathrm{H}$, pyrazole- $\left.\mathrm{CH}_{3}\right), 4.78\left(\mathrm{~d}, J=8.0 \mathrm{~Hz}, 2 \mathrm{H}, \mathrm{CH}_{2}\right), 6.48(\mathrm{~s}, 1 \mathrm{H}$, isoxazole-H), $6.54(\mathrm{~s}, 1 \mathrm{H}, \mathrm{CONH}), 6.89(\mathrm{t}, J=36.0 \mathrm{~Hz}$, $\left.1 \mathrm{H}, \mathrm{CHF}_{2}\right), 7.23 \sim 7.36(\mathrm{~m}, 3 \mathrm{H}, \operatorname{Ar}-\mathrm{H}), 7.97(\mathrm{~s}, 1 \mathrm{H}$, pyrazole-H); MS (ESI) $m / z$ : $385[\mathrm{M}+\mathrm{H}]^{+}$. HRMS calcd for $\mathrm{C}_{16} \mathrm{H}_{12} \mathrm{ClF}_{3} \mathrm{~N}_{4} \mathrm{O}_{2}$ 385.0674, found 385.0688.

3 -二氟甲基- $N$ - $[(3-(2,6-$ 二氟苯基)异噁唑-5-基)-甲 基]-1-甲基- $1 H$-吡唑-4-甲酰胺(12h): 黄色油状液体, 产 率 77.1\%. ${ }^{1} \mathrm{H}$ NMR $\left(\mathrm{CDCl}_{3}, 400 \mathrm{MHz}\right) \delta: 3.94(\mathrm{~s}, 3 \mathrm{H}$, pyrazole- $\left.\mathrm{CH}_{3}\right), 4.78\left(\mathrm{~d}, J=8.0 \mathrm{~Hz}, 2 \mathrm{H}, \mathrm{CH}_{2}\right), 6.48(\mathrm{~s}, 1 \mathrm{H}$, isoxazole-H), $6.54(\mathrm{~s}, 1 \mathrm{H}, \mathrm{CONH}), 6.89(\mathrm{t}, J=36.0 \mathrm{~Hz}$, $\left.1 \mathrm{H}, \mathrm{CHF}_{2}\right), 7.23 \sim 7.36(\mathrm{~m}, 3 \mathrm{H}, \operatorname{Ar}-\mathrm{H}), 7.97(\mathrm{~s}, 1 \mathrm{H}$, pyrazole-H); MS (ESI) $\mathrm{m} / z: 369[\mathrm{M}+\mathrm{H}]^{+}$. HRMS calcd for $\mathrm{C}_{16} \mathrm{H}_{12} \mathrm{~F}_{4} \mathrm{~N}_{4} \mathrm{O}_{2} 369.0969$, found 369.0987 .

3-二氟甲基- $N$ - $[(3-$-(2,3,4-三甲氧基苯基)异噁唑-5基)-甲基]-1-甲基- $1 H$-吡唑-4-甲酰胺(12i): 棕色油状液 体, 产率 45.67\%. ${ }^{1} \mathrm{H}$ NMR $\left(\mathrm{CDCl}_{3}, 400 \mathrm{MHz}\right) \delta: 3.83$ (s, $\left.9 \mathrm{H}, \mathrm{OCH}_{3}\right), 3.94\left(\mathrm{~s}, 3 \mathrm{H}\right.$, pyrazole- $\left.\mathrm{CH}_{3}\right), 4.71(\mathrm{~d}, J=8.0$ $\left.\mathrm{Hz}, 2 \mathrm{H}, \mathrm{CH}_{2}\right), 6.48(\mathrm{~s}, 1 \mathrm{H}$, isoxazole-H), $6.55(\mathrm{~s}, 1 \mathrm{H}$, $\mathrm{CONH}), 6.88\left(\mathrm{~d}, J=36.0 \mathrm{~Hz}, 1 \mathrm{H}, \mathrm{CHF}_{2}\right), 6.79 \sim 6.99(\mathrm{~m}$, 2H, Ar-H), 7.96 (s, 1H, pyrazole-H); MS (ESI) $m / z$ : 423 $[\mathrm{M}+\mathrm{H}]^{+}$. HRMS calcd for $\mathrm{C}_{19} \mathrm{H}_{20} \mathrm{~F}_{2} \mathrm{~N}_{4} \mathrm{O}_{5} 423.1475$, found 423.1486 .

3 -二氟甲基- $N$-[(3-(2,6-二甲氧基苯基)异噁唑-5基)-甲基]-1-甲基- $1 \mathrm{H}$-吡唑-4-甲酰胺(12j)：淡黄色油状 液体, 产率 $37.7 \%$. ${ }^{1} \mathrm{H} \mathrm{NMR}\left(\mathrm{CDCl}_{3}, 400 \mathrm{MHz}\right) \delta: 3.77$ (s, $\left.6 \mathrm{H}, \mathrm{OCH}_{3}\right), 3.92\left(\mathrm{~s}, 3 \mathrm{H}\right.$, pyrazole- $\left.\mathrm{CH}_{3}\right), 4.80(\mathrm{~d}, J=8.0$ $\left.\mathrm{Hz}, 2 \mathrm{H}, \mathrm{CH}_{2}\right), 6.37$ (s, $1 \mathrm{H}$, isoxazole-H), 6.70 (d, $J=8.0$ $\mathrm{Hz}, 2 \mathrm{H}, \mathrm{Ar}-\mathrm{H}), 6.89$ (d, J=36.0 Hz, 1H, $\mathrm{CHF}_{2}$ ), 6.92 (s, 1H, CONH), 7.40 (s, 1H, Ar-H), 7.96 (s, 1H, pyrazole-H); MS (ESI) $m / z: 393[\mathrm{M}+\mathrm{H}]^{+}$. HRMS calcd for $\mathrm{C}_{18} \mathrm{H}_{18} \mathrm{~F}_{2-}$ $\mathrm{N}_{4} \mathrm{O}_{4} 393.1369$, found 393.1386.

3-二氟甲基- $N$-[(3-(2-羟基苯基)异噁唑-5-基)-甲 
基]-1-甲基-1 $H$-吡唑-4-甲酰胺(12k): 棕色油状液体，产 率 71.0. ${ }^{1} \mathrm{H} \mathrm{NMR}\left(\mathrm{CDCl}_{3}, 400 \mathrm{MHz}\right) \delta$ : $3.94(\mathrm{~s}, 3 \mathrm{H}$, pyrazole- $\left.\mathrm{CH}_{3}\right), 4.79\left(\mathrm{~d}, J=8.0 \mathrm{~Hz}, 2 \mathrm{H}, \mathrm{CH}_{2}\right), 6.66(\mathrm{~s}, 1 \mathrm{H}$, isoxazole-H), $6.90\left(\mathrm{~d}, J=36.0 \mathrm{~Hz}, 1 \mathrm{H}, \mathrm{CHF}_{2}\right), 6.91 \sim 6.99$ (m, 2H, Ar-H and CONH), 7.33 (t, $J=8.0 \mathrm{~Hz}, 1 \mathrm{H}, \mathrm{Ar}-\mathrm{H})$, 7.46 (d, $J=8.0 \mathrm{~Hz}, 1 \mathrm{H}, \operatorname{Ar}-\mathrm{H}), 7.95$ (s, 1H, OH), 8.01 (s, $1 \mathrm{H}$, pyrazole-H); MS (ESI) $m / z: 349[\mathrm{M}+\mathrm{H}]^{+}$. HRMS calcd for $\mathrm{C}_{16} \mathrm{H}_{14} \mathrm{~F}_{2} \mathrm{~N}_{4} \mathrm{O}_{3} 349.1107$, found 349.1110.

3-二氟甲基- $N$ - [(3-(4-叔丁基苯基)异啞唑-5-基)-甲 基]-1-甲基- $1 H$-吡唑-4-甲酰胺(12l)：黄色油状液体，产 率 89.18\%. ${ }^{1} \mathrm{H} \mathrm{NMR}\left(\mathrm{CDCl}_{3}, 400 \mathrm{MHz}\right) \delta: 2.17$ (s, 9H, $\left.\mathrm{CH}_{3}\right), 3.93$ (s, 3H, pyrazole- $\left.\mathrm{CH}_{3}\right), 4.76(\mathrm{~d}, J=8.0 \mathrm{~Hz}, 2 \mathrm{H}$, $\left.\mathrm{CH}_{2}\right), 6.52$ (s, 1H, isoxazole-H), 6.55 (s, 1H, CONH), 6.90 (d, $\left.J=36.0 \mathrm{~Hz}, 1 \mathrm{H}, \mathrm{CHF}_{2}\right), 7.46$ (d, $\left.J=8.0 \mathrm{~Hz}, 1 \mathrm{H}, \mathrm{Ar}-\mathrm{H}\right)$, 7.71 (d, $J=8.0 \mathrm{~Hz}, 1 \mathrm{H}$, Ar-H), 7.97 (s, 1H, pyrazole-H); MS (ESI) $m / z: 389[\mathrm{M}+\mathrm{H}]^{+}$. HRMS calcd for $\mathrm{C}_{20} \mathrm{H}_{22} \mathrm{~F}_{2^{-}}$ $\mathrm{N}_{4} \mathrm{O}_{2} 389.1784$, found 389.1782.

3 -二氟甲基- $N$-[(3-(3-甲基噻吩-2-基)异噁唑-5-基)甲基]-1-甲基- $1 H$-吡唑-4-甲酰胺(12m): 棕色油状液体, 产率 63.4\%. ${ }^{1} \mathrm{H} \mathrm{NMR}\left(\mathrm{CDCl}_{3}, 400 \mathrm{MHz}\right) \delta: 2.45(\mathrm{~s}, 3 \mathrm{H}$, thiophene- $\left.\mathrm{CH}_{3}\right), 3.94\left(\mathrm{~s}, 3 \mathrm{H}\right.$, pyrazole- $\left.\mathrm{CH}_{3}\right), 4.76(\mathrm{~d}, J=$ $\left.8.0 \mathrm{~Hz}, 2 \mathrm{H}, \mathrm{CH}_{2}\right), 6.45$ (s, $1 \mathrm{H}$, isoxazole-H), 6.89 (d, $J=$ $\left.36.0 \mathrm{~Hz}, 1 \mathrm{H}, \mathrm{CHF}_{2}\right), 6.93(\mathrm{~d}, J=8.0 \mathrm{~Hz}, 1 \mathrm{H}$, thiophene-H), $7.29(\mathrm{~d}, J=8.0 \mathrm{~Hz}, 1 \mathrm{H}$, thiophene-H), 7.98 (s, 1H, pyrazole-H); MS (ESI) m/z: $353[\mathrm{M}+\mathrm{H}]^{+}$. HRMS calcd for $\mathrm{C}_{15} \mathrm{H}_{14} \mathrm{~F}_{2} \mathrm{~N}_{4} \mathrm{O}_{2} \mathrm{~S} 353.0878$, found 353.0882.

辅助材料(Supporting Information) 13 个目标化合物 的质谱分析图. 这些材料可以免费从本刊网站 (http://sioc-journal.cn/)上下载.

\section{References}

[1] Liu, X. H.; Zhao, W. G.; Wang, B. L.; Li, Z. M. Res. Chem. Intermed. 2012, 28, 1999.

[2] Tong, J. Y.; Shi, Y. X.; Liu, X. H.; Sun, N. B.; Li, B. J. Chin. J. Org. Chem. 2012, 32, 2373 (in Chinese).

(童建颖, 石延霞, 刘幸海, 孙娜波, 李宝聚, 有机化学, 2012,
32, 2373.)

[3] Su, N. N.; Li, Y.; Yu, S. J.; Zhang, X.; Liu, X. H.; Zhao, W. G. Res. Chem. Intermed. 2013, 39, 759.

[4] Tong, J. Y.; Wu, H. K.; Sun, N. B.; Liu, X. H. Chin. J. Struct. Chem. 2013, 32, 607.

[5] Hu, L.-M.; Li, X.-S.; Chen, Z.-Y.; Liu, Z.-J. Chin. J. Org. Chem. 2003, 23, 1131 (in Chinese).

(胡利明, 李学恕, 陈致远, 刘钊杰, 有机化学, 2003, 23, 1131.)

[6] (a) Xe, F.; Liu, T.-T.; Yang, G.; Yuan, J.; Kong, X.-L.; Xu, T.-M.; Tan, C.-X. Chin. J. Org. Chem. 2013, 33, 2596 (in Chinese). (谢峰, 刘婷婷, 杨果, 袁静, 孔小林, 许天明, 谭成侠, 有机化 学, 2013, 33, 2596.)

(b) Ma, Q.-S.; Liu, X.-H.; Weng, J.-Q.; Li, Y.-S.; Zhang, M.; Zhang, X.-Y.; Tan, C.-X. Chin. J. Org. Chem. 2013, 33, 1749 (in Chinese).

(马青森, 刘幸海, 翁建全, 李永曙, 张旻, 张向阳, 谭成侠, 有 机化学, 2013, 33, 1749.)

[7] Ohuchi, S.; Okada, S. JP 10338589, 1998 [Chem. Abstr. 1998, 130, 120912y].

[8] Liu, C.-L. Pesticide 1998, 37(8), 1 (in Chinese). (刘长令, 农药, 1998, 37(8), 1.)

[9] Doyle, F. P.; J. C.; Long, A. A. W.; Nayler, J. H. C.; Stove, E. R. J. Am. Chem. Soc. 1963, 85, 5845.

[10] Kang, Y. K.; Shin, K. J.; Yoo, K. H.; Seo, K. J.; Hong, C. Y.; Lee, C. S.; Park, S. Y.; Kim, D. T.; Park, S. W. Bioorg. Med. Chem. Lett. 2000, 10, 95.

[11] Josef, E.; Harald, W.; Camilla, C.; Hans, T. WO 2007065661, 2007 [Chem. Abstr. 2007, 147, 72747].

[12] Dunkel, R.; Beeck, S.; Greul, J. N.; Wachendorf-Neumann, U.; Dahmen, P.; Voerste, A. WO 2008014905, 2008 [Chem. Abstr. 2008, 148, 239197].

[13] Stierli, D.; Taylor, J. J.; Walter, H.; Worthington, P. A.; Rajan, R. WO 2007141009, 2007 [Chem. Abstr. 2007, 148, 55044].

[14] Stierli, D.; Taylor, J. J.; Walter, H.; Worthington, P. A. WO 2007134799, 2008 [Chem. Abstr. 2008, 148, 168714].

[15] EP 1997808, 2008 [Chem. Abstr. 2008, 150, 5730].

[16] (a) Du, G.-J.; Chen, D.-L.; Lu, R.-J.; Wang, X.-J.; Yan, M. Chin. J. Org. Chem. 2009, 29, 1576 (in Chinese).

(杜光剑, 陈东亮, 卢锐昫, 王晓军, 鄢明, 有机化学, 2009, 29, 1576.)

(b) Zhou, Y.-L.; Shen, S.-W.; Liu, F.-M. Chin. J. Org. Chem. 2010, 30, 1343 (in Chinese).

(周英雷, 沈松伟, 刘方明, 有机化学, 2010, 30, 1343.)

[17] Hu, W.-Q.; Zhu, W.-G.; Chen, D.-H. Chin. J. Pestic. Sci. 2007, 9, 240 (in Chinese).

(胡伟群, 朱卫刚, 陈定花, 农药学学报, 2007, 9, 240.)

[18] Stierli, D.; Walter, H.; Rajan, R. WO 2009127726, 2009 [Chem. Abstr. 2009, 151, 470195].

(Qin, X.) 